Cite this: J. Mater. Chem. A, 2014, 2, 10193

\author{
Received 20th March 2014 \\ Accepted 13th May 2014 \\ DOI: $10.1039 / c 4 t a 01373 e$ \\ www.rsc.org/MaterialsA
}

\section{Colloidal cubes for the enhanced degradation of organic dyes $\dagger$}

\author{
Sonja I. R. Castillo, ${ }^{a}$ Cornelia E. Pompe, ${ }^{a}$ Jim van Mourik, ${ }^{a}$ David M. A. Verbart, ${ }^{a}$ \\ Dominique M. E. Thies-Weesie, ${ }^{a}$ Petra E. de Jongh ${ }^{b}$ and Albert P. Philipse ${ }^{* a}$
}

\begin{abstract}
The shape of cubic silica colloids is exploited to form close-packed structures, whereas the hollow core of the cubes may host functional substances. A proof-of-principle of this approach is presented for iron oxide particles (hematite, $\boldsymbol{\alpha}-\mathrm{Fe}_{2} \mathrm{O}_{3}$ ) confined inside silica cubes that display the ability to accelerate the degradation of the organic dyes methylene blue and rhodamine $B$ in the presence of hydrogen peroxide (modified Fenton reaction). The silica coating does not impede the reaction, since degradation rates similar to those of bare hematite particles are observed. Moreover, the cubic colloids are still functional when densely packed onto a substrate. The degradation reaction is greatly enhanced by illumination with visible or UV light: the degradation time reduces by two orders of magnitude. Although the silica coating is damaged during the degradation reaction, the stability of the coating is improved by heat treatment of the cubes and by illumination.
\end{abstract}

\section{Introduction}

Core-shell particles have been extensively studied for their ability to combine distinct features of individual systems into one system. Particularly, a great body of literature focuses on (nano)particles as functional materials encapsulated by a protecting shell. ${ }^{1-7}$ Typical systems are silver or gold nanoparticles coated with silica, since these nanoparticles are catalytically active..$^{8-12}$ Generally, the functional particles are spherical in shape, resulting in spherical core-shell particles. Although functional core-shell particles are often utilized as freely dispersed particles, immobilization on a substrate is clearly of interest for many applications, e.g., separation membranes and coatings. Ideally, the substrate has a high packing density. Spheres randomly pack to a solid volume fraction of $\phi \approx 0.63$; one option to increase this packing density is to employ nonspherical particles in the form of cubes. Recently developed colloidal silica cubes are indeed able to form dense assemblies of close-packed cubes. ${ }^{13,14}$ Computational studies of randomly packed particles with the shape of these colloidal silica cubes, i.e., cubes with rounded edges, show that a solid volume fraction between 0.71 and 0.74 can be obtained, depending on the roundedness of the cubes. ${ }^{15,16}$ The silica cubes are synthesized by coating a cubic shaped iron oxide (hematite, $\alpha-\mathrm{Fe}_{2} \mathrm{O}_{3}$ ) core

${ }^{a}$ Van 't Hoff Laboratory for Physical and Colloid Chemistry, Debye Institute for Nanomaterials Science, Utrecht University, Padualaan 8, 3584 CH, Utrecht, The Netherlands. E-mail: a.p.philipse@uu.nl; Fax: +31 30 2533870; Tel: + 31302533518 ${ }^{b}$ Inorganic Chemistry and Catalysis, Debye Institute for Nanomaterials Science, Utrecht University, Universiteitsweg 99, 3584 CG, Utrecht, The Netherlands

$\dagger$ Electronic supplementary information (ESI) available. See DOI: 10.1039/c4ta01373e with amorphous silica. ${ }^{13,17}$ In this study, the hematite core is exploited to accelerate the degradation of organic dyes, while the cubic shape is maintained by the silica shell to form closepacked assemblies of these functional particles.

The enhanced degradation of organic dyes, often found in waste water of the textile industry, ${ }^{18}$ is achieved using Fenton's reagent: hydrogen peroxide in combination with an iron ion source. ${ }^{18-24}$ The iron ion source promotes the formation of hydroxyl radicals $\left(\mathrm{OH}^{*}\right)$, which readily degrade organic compounds, according to the following mechanism ${ }^{22,25-28}$

$$
\begin{gathered}
\mathrm{Fe}^{2+}+\mathrm{H}_{2} \mathrm{O}_{2} \rightarrow \mathrm{Fe}^{3+}+\mathrm{OH}^{\cdot}+\mathrm{OH}^{-} \\
\mathrm{Fe}^{3+}+\mathrm{H}_{2} \mathrm{O}_{2} \rightarrow \mathrm{Fe}^{2+}+\mathrm{OOH}^{\cdot}+\mathrm{H}^{+} \\
\mathrm{Fe}^{3+}+\mathrm{OOH}^{\cdot} \rightarrow \mathrm{Fe}^{2+}+\mathrm{O}_{2}+\mathrm{H}^{+} \\
\mathrm{OH}^{\cdot}+\mathrm{H}_{2} \mathrm{O}_{2} \rightarrow \mathrm{OOH}^{\cdot}+\mathrm{H}_{2} \mathrm{O} \\
\mathrm{OH}^{\cdot}+\text { organic molecule } \rightarrow \text { fragments }
\end{gathered}
$$

This process is favored by acidic conditions, with an optimum at $\mathrm{pH} 3$, and is further accelerated by illumination with visible or UV light (photo-Fenton reaction). ${ }^{23-25}$

In our study, the hematite cores of the cubic colloids serve as the iron source. Iron oxide particles as the iron source for the Fenton reaction are favorable because of their easy recovery and the absence of iron sludge formation otherwise observed with dissolved iron ions as iron source. ${ }^{18,22,24,25}$ Since in the classic Fenton reaction iron salts are used, we refer to the iron oxide particles based reactions as modified Fenton reactions. ${ }^{19}$ Although other iron oxides have been shown to be more 
efficient in the modified Fenton reaction, ${ }^{\mathbf{2 0 - 2 2}}$ hematite is an appealing material due to its thermodynamic stability, abundance and its ability to absorb a large part of the solar spectrum..$^{29-33}$ For these reasons, it is also widely investigated as a photocatalyst for low-coast water splitting. ${ }^{29-32}$ Hematite particles with a well-defined shape applied in the modified Fenton reaction other than our colloidal cubes include amongst others, colloidal hollow polyhedra thin shells, ${ }^{34}$ colloidal spindels, ${ }^{20}$ nanorhombohedra ${ }^{35}$ and nanocubes, nanoplatelets and (nano) rods. ${ }^{36,37}$ Commonly, the influence of the exposed facets on the catalytic properties of the particles is investigated. Compared to most of the mentioned hematite particles, our cubic colloids are advantageous due to the high packing density they can achieve resulting from their shape. ${ }^{\mathbf{1 5 1 6}}$ This property is especially appealing for immobilizing the functional particles on substrates. Moreover, the silica shell allows for the preservation of the cubic shape while the surface area of the hematite core can be changed by selective acid treatment without affecting the silica shell. In this study we demonstrate that the colloidal cubes are not only of interest for fundamental phase behavior studies, but can also be employed in functional materials by combining their dense packing behavior with their catalytic properties.

This paper comprises the experimental details and results of both the synthesis of the colloidal cubes as well as their use in dye degradation experiments. For the degradation experiments, the cubes are either freely dispersed in the reaction mixture or deposited on a substrate. Additionally, the stability of the cubes during the reaction is discussed.

\section{Experimental}

\subsection{Particle synthesis}

2.1.1 Hematite cubes. Hematite $\left(\alpha-\mathrm{Fe}_{2} \mathrm{O}_{3}\right)$ cubic particles were synthesized following the adjusted method of Sugimoto et al. as described in detail in the ESI. $\dagger^{\mathbf{1 3 , 3 8 , 3 9}}$ In this synthesis, aqueous solutions of sodium hydroxide (p.a., Emsure) and iron(III) chloride (iron(III) chloride hexahydrate, p.a., SigmaAldrich) are mixed to form a gel. The gel is aged at $100{ }^{\circ} \mathrm{C}$ for eight days, during which it transforms from the gel to akaganéite needles and eventually to a sol of hematite cubes. The edge length of the hematite cubes can be varied between $500 \mathrm{~nm}$ and $1500 \mathrm{~nm}$ by adjusting the concentration of excess ferric ions. An increased concentration of base leads to smaller particles due to a decreased amount of ferric ions available for particle growth. ${ }^{38}$ The precise reactant amounts and concentrations of excess ferric ions for each experiment are listed in the ESI. $\dagger$

2.1.2 Silica coated hematite cubes. Hematite cubes were coated with Stöber silica according to the method described by Rossi et al. ${ }^{13,17}$ To coat the cubes with a smooth and uniform layer of silica, functionalization of the cubic seed particles with the polymer polyvinylpyrrolidone (PVP, $40 \mathrm{~kg} \mathrm{~mol}^{-1}$, SigmaAldrich) is first needed. Amorphous silica is then grown by slowly adding the silica precursor tetraethyl orthosilicate (TEOS, puriss $\geq 99.0 \%$, Sigma-Aldrich) to the cubes in a mixture of ethanol (100\%, Interchema) and Millipore water. This reaction is catalyzed by the base tetramethylammonium hydroxide (TMAH, $25 \mathrm{wt} \%$ in water, Fluka). The thickness of the coating is tuned by varying the amount TEOS added. Typically, $10 \mathrm{~mL}$ TEOS diluted to $20 \mathrm{~mL}$ with ethanol was used to obtain a coating of approximately $80 \mathrm{~nm}$ in thickness onto a batch of $1 \mathrm{~g}$ hematite cubes. The entire reaction should be conducted under mechanical stirring and ultrasonication at a constant temperature of $20{ }^{\circ} \mathrm{C}$ to prevent aggregation of the hematite cubes during synthesis. In order to grow a silica coating of the same thickness on a larger amount of seed particles, multiple growth steps are needed. To coat $1.8 \mathrm{~g}$ of hematite cubes, three growth steps were performed.

2.1.3 Etching of the hematite core. The hematite core was etched to modify its surface area, since a higher surface area results in an increased amount of accessible iron ions for the reaction. For the etching process, an aqueous solution of hydrochloric acid (6-12 M, Emsure) was added in portions to the silica coated hematite cubes dispersed in water. Since the silica coating is porous, the acid can access the hematite core and the ions from the dissolved hematite can diffuse away to the surrounding water. The etched cubes were then washed by centrifugation and redispersion until $\mathrm{pH}$ 6-7 was reached. The silica coating is not affected by the acid treatment, so the cubic shape is maintained while the hematite core is modified. These etched particles are referred to as rattles onward, due to the rapid motion in an enclosed space resembling the kids' toy or percussion instrument.

\subsection{Degradation experiments}

To determine the catalytic activity of the cubes, degradation experiments of organic dyes were conducted. The reaction mixtures were prepared in $20 \mathrm{~mL}$ glass vials. First, $5 \mathrm{~mL}$ of aqueous solutions of either methylene blue $\left(4.4 \times 10^{-4} \mathrm{M}, \mathrm{J}\right.$. T. Baker) or rhodamine B $\left(1.82 \times 10^{-4} \mathrm{M}\right.$, Sigma-Aldrich) was brought in the vial. Second, the cubes were inserted, either as freely dispersed particles (typically $0.01 \mathrm{~g}$ dry weight) or as substrated particles (typically $0.02 \mathrm{~g}$ dry weight). Finally, $5 \mathrm{~mL}$ $35 \mathrm{wt} \% \mathrm{H}_{2} \mathrm{O}_{2}$ (35 wt\% solution in water, stabilized, p.a., Acros Organics) was added and the total volume was brought to $20 \mathrm{~mL}$ with Millipore water. The initial $\mathrm{pH}$ of the reaction mixtures was pH 3, caused by hydrogen peroxide. The pH was not buffered during the reaction, but the $\mathrm{pH}$ after the reaction was still approximately 3 .

The experiments were performed at either regular laboratory lighting or under active illumination of visible light $(\lambda>420 \mathrm{~nm}$, incandescent light bulb $230 \mathrm{~V}, 40 \mathrm{~W})$ or UV light $(\lambda=365 \mathrm{~nm}$, Sylvania F8T5/BLB, $8 \mathrm{~W}$ ). The lamps were placed at a distance of approximately $30 \mathrm{~cm}$ from the reaction vials and the light was reflected from the back to enhance illumination. For the cubes on a substrate, only regular laboratory lighting was used.

2.2.1 Dispersed cubes. The dispersed cubes (typically $0.01 \mathrm{~g}$ dry weight) were stirred magnetically during the reaction and the lids of the vials were screwed loosely.

2.2.2 Cubes on a substrate. For the experiments with cubes on a mica substrate, cubes dispersed in water were deposited on the substrates with a Pasteur pipette until a large drop was 
formed. The deposition was done twice, so a sufficient amount of particles was present (typically $0.02 \mathrm{~g}$ dry weight). The solvent was evaporated completely before applying a heat treatment. Heat treatment ( 3 hours at $450{ }^{\circ} \mathrm{C}$, heat increment and decrement $\sim 45{ }^{\circ} \mathrm{C} \mathrm{h}^{-1}$, Nabertherm N15/65 HA oven) after the deposition steps is needed to prevent the cubes from releasing from the substrate too easily. Mica was chosen as the substrate since it is heat resistant and atomically flat after cleaving.

For degradation experiments with cubes on substrates, two different setups were utilized. In the first setup, the mica substrate $(4 \times 2 \mathrm{~cm})$ is hung vertically in the reaction mixture, which is stirred magnetically. For the second setup, the substrate $(2 \times 2 \mathrm{~cm})$ is placed horizontally on the bottom of a $100 \mathrm{~mL}$ vial with the cubes on the top side and the reaction mixture is stirred slowly with an overhead stirrer. The advantage of this setup over the former is that it ensures that all cubes are in the reaction mixture. Moreover, the detachment of the cubes from the substrate is less likely, since the substrate is not disturbed by the stirrer.

\subsection{Characterization}

\subsubsection{Microscopy}

2.3.1.1 Optical microscopy. For the visualization of particles with an optical microscope, a Nikon eclipse Ti inverse optical microscope with a $100 \times$ Nikon oil immersion objective and an InfinityX CCD camera was used. Small drops $(\sim 20 \mu \mathrm{L})$ were placed on a glass microscope slide.

2.3.1.2 Electron microscopy. Transmission Electron Microscopy (TEM) images were taken with a Philips TECNAI10 or TECNAI12, which have a maximum acceleration voltage of $100 \mathrm{kV}$ and $120 \mathrm{kV}$, respectively. Samples were prepared by dropcasting a liquid sample onto a Formvar and carbon coated copper TEM grid and were dried under a heating lamp.

To visualize the substrates with Scanning Electron Microscopy (SEM), a Philips XLFEG30 was used with a maximum acceleration speed of $10 \mathrm{kV}$. The substrates were placed on a carbon sticker on a SEM stub prior to sputter coating with a platinum layer of $6 \mathrm{~nm}$.

2.3.2 Nitrogen physisorption. Nitrogen physisorption measurements on the colloidal cubes, both etched and unetched, were performed on a Micromeritics TriStar 3000 to determine the surface areas. The cubes were dried in a sample concentrator at $70{ }^{\circ} \mathrm{C}$ under a nitrogen gas flow for one or two days. These dry samples were dried further overnight at $180{ }^{\circ} \mathrm{C}$ under a nitrogen gas flow with a Micromeritics Smartprep prior to actual measurement. The specific surface area is derived by fitting the BET-isotherm from $p / p_{0}=0.06$ to $p / p_{0}=0.25$ of the measured adsorption isotherm.

2.3.3 UV-vis spectroscopy. For the degradation reaction, methylene blue and rhodamine $\mathrm{B}$ were employed as model compounds. All spectra were recorded on a Perkin-Elmer Lambda 35 UV-vis spectrometer from $\lambda=700$ to $\lambda=200 \mathrm{~nm}$. Hellma 110-QS quartz cuvettes with a path length of $10 \mathrm{~mm}$ were used. The peaks of the methylene blue and rhodamine B in the visible range are used for analysis, since the spectra of the organic molecules and hydrogen peroxide overlap in the UV range and hydrogen peroxide could not be removed from the sample prior to measurement. The absorbance at $\lambda=609 \mathrm{~nm}$ was measured to monitor the temporal concentration of methylene blue, since the peak around $668 \mathrm{~nm}$ is concentration dependent. The molar extinction coefficient for the peak at $\lambda=$ $609 \mathrm{~nm}$ was determined to be $33419 \mathrm{M}^{-1} \mathrm{~cm}^{-1}$. For rhodamine $\mathrm{B}$, the peak at $\lambda=554 \mathrm{~nm}$ is used to monitor the concentration change over time. The molar extinction coefficient was determined to be $101426 \mathrm{M}^{-1} \mathrm{~cm}^{-1}$.

2.3.3.1 Sample preparation. To obtain a suitable sample for the UV-vis measurements, reaction mixtures were centrifuged to remove the cubes, which absorb and scatter light rendering the measurements unreliable. Removal of the cubes by means of filtration of the reaction mixture using filters with $100 \mathrm{~nm}$ size filter holes was not suitable because the filters absorbed an unknown amount of dye.

2.3.4 Infrared spectroscopy. Infrared (IR) spectroscopy was employed to monitor the extent of hematite etching, using the silica peak as an internal standard. The peak of hematite is at approximately $550 \mathrm{~cm}^{-1}$ and the peak of silica is around $1100 \mathrm{~cm}^{-1}$ (Fig. S3 in the ESI $\dagger$ ). ${ }^{33,40,41}$ The spectra were recorded using potassium bromide (KBr, p.a. for IR spectroscopy, Fluka) pellets as medium.

2.3.5 $\mathrm{pH}$ measurements. The $\mathrm{pH}$ was measured with $\mathrm{pH}$ paper (Merck, Universal indicator, $\mathrm{pH}$ 0-14) or a Hanna instruments $\mathrm{pH} 210$ microprocessor $\mathrm{pH}$ meter. The $\mathrm{pH}$ meter was calibrated at $\mathrm{pH} 4$ and 7.

\section{Results and discussion}

\subsection{Particle synthesis}

To determine the effect of the size of the cubic colloids on the degradation rates, we synthesized hematite cubes of different sizes. Table 1 lists the cubic colloids used in this research. The bare hematite cubes are labeled with $\mathrm{H}$ and the corresponding silica coated hematite cubes are labeled with S. Representative TEM images of these cubes are shown in Fig. 1 and in the ESI. $\dagger$

Generally, the cubic shape is better defined and the polydispersity is lower with increasing size. The shape of the small

Table 1 Properties of the cubes used in this research. The given sizes are the average edge lengths and pd\% is the polydispersity. Cubes marked by an * were later on subjected to hematite etching. For the cubes of $\mathrm{S} 4 \mathrm{a}$, three silica coating procedures were performed to obtain the final size

\begin{tabular}{lllll}
\hline Cubes & $\begin{array}{l}\text { Core size } \\
(\mathrm{nm})\end{array}$ & $\begin{array}{l}\text { Final size } \\
(\mathrm{nm})\end{array}$ & $\begin{array}{l}\text { pd\% } \\
(\%)\end{array}$ & $\begin{array}{l}\text { Thickness } \\
\text { silica (nm) }\end{array}$ \\
\hline $\mathrm{H} 1$ & 374 & & 14 & \\
$\mathrm{H} 2$ & 663 & & 7 & \\
$\mathrm{H} 3$ & 800 & & 5 & \\
$\mathrm{H} 4 *$ & 920 & & 7 & \\
S1 & 374 & 431 & 12 & 30 \\
S2 & 663 & 813 & 4 & 75 \\
S3 & 800 & 924 & 4 & 65 \\
S4a* & 920 & 1070 & 8 & 75 \\
S4b & 920 & 1093 & 6 & 85
\end{tabular}




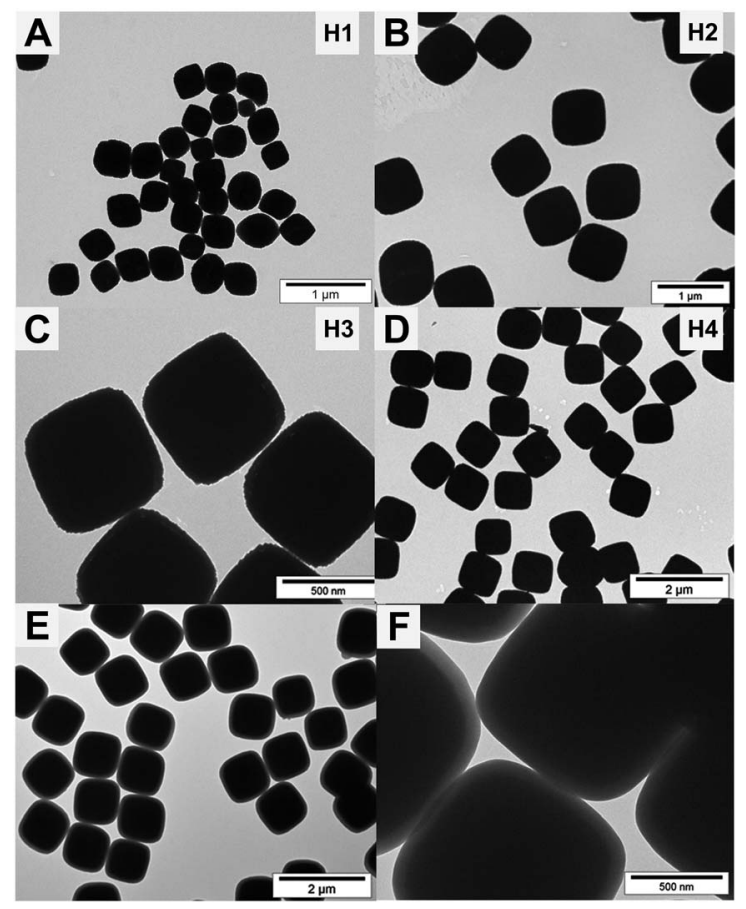

Fig. 1 Typical TEM images of the cubic colloids used in this research. Their properties are listed in Table 1. The first four images are of bare hematite cubes with an average edge length of (A) $374 \mathrm{~nm}(\mathrm{H1})$, (B) 663 $\mathrm{nm}(\mathrm{H} 2),(\mathrm{C}) 800 \mathrm{~nm}(\mathrm{H} 3)$ and (D) $920 \mathrm{~nm}(\mathrm{H} 4)$, respectively. The cubic shape is less pronounced for the smaller particles. (E) and (F) Low and high magnification images of silica coated hematite cubes. All silica coatings are smooth and uniform.

particles (average size of $374 \mathrm{~nm}$ ) deviates slightly from the cubic shape, i.e., two opposite corners are more elongated resulting in a walnut-like shape. The obtained silica coatings are all smooth and uniform. The cubes that were silica coated in a three step procedure (S4a) are shown in Fig. 1E and have a silica coating with a final thickness of $75 \mathrm{~nm}$.

\subsubsection{Hematite etching}

3.1.1.1 Bare cubes. To investigate the effect of hematite etching on the surface area of the particles, bare cubes (H4) etched to different degrees were prepared. The extent of etching was followed conveniently with optical microscopy, with which the morphology change could be clearly observed (Fig. 2 top row). However, the final morphologies were determined more precisely with TEM (Fig. 2 bottom row). As the etching proceeds, the characteristic butterfly shape of etched cubes emerges. ${ }^{42}$

The remaining hematite was weighed after drying. For the etched particles in Fig. 2B, 25\% of the initial mass of hematite was left and only $1 \%$ for the particles in Fig. $2 \mathrm{C}$.

The BET-surface areas are listed in Table 2 and as expected, the change in morphology to butterfly-like particles results in a higher surface area and a more porous structure (see ESI $\dagger$ ). However, prolonged etching changes the morphology of the iron oxide particles such that the surface area and porosity decrease again. Apparently, a maximum can be found between 25 and $1 \mathrm{wt} \%$ of remaining hematite.

The BET-surface area determined for the unetched particles, i.e., the entirely cubic particles, is notably higher than expected

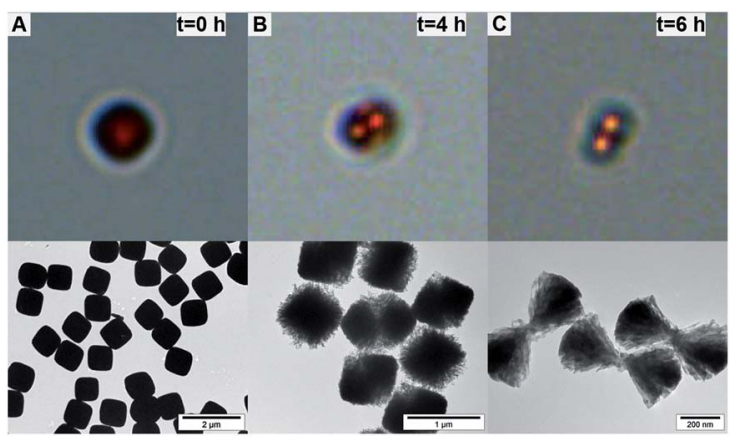

Fig. 2 Microscope images (top row: optical microscopy; bottom row: electron microscopy) of hematite etched particles followed over time. Optical microscopy is a fast method to examine the shape of the etched cubes, but the precise morphology needs to be determined with TEM. The shape of the particle clearly changes as etching proceeds. (A) Reference image of an unetched bare hematite cube (H4). (B) Hematite cube etched for four hours, 25 wt\% of the initial amount of hematite remaining. (C) Hematite cube etched for six hours, $1 \mathrm{wt} \%$ of the initial amount of hematite remaining.

Table 2 The total BET-surface areas $S_{\text {total }}$ for cubes subjected to hematite etching at increasing extent. For silica coated cubes, also the mass fraction of the silica shell and the calculated contribution of the silica shell to the total BET-surface area are listed. Calculations are explained in the text. The resulting surface areas of the hematite cores are given in the last column

\begin{tabular}{lcclll}
\hline & $\begin{array}{l}\text { Remaining } \\
\text { hematite } \\
(\mathrm{wt} \%)\end{array}$ & $\begin{array}{l}S_{\mathrm{total}} \\
\left(\mathrm{m}^{2} \mathrm{~g}^{-1}\right)\end{array}$ & $\begin{array}{l}\text { Mass } \\
\text { fraction } \\
\mathrm{SiO}_{2}\end{array}$ & $\begin{array}{l}S_{\mathrm{SiO}_{2}}: \\
S_{\text {total }}(\%)\end{array}$ & $\begin{array}{l}S_{\alpha-\mathrm{Fe}_{2} \mathrm{O}_{3}} \\
\left(\mathrm{~m}^{2} \mathrm{~g}^{-1}\right)\end{array}$ \\
\hline H4 unetched & 100 & 35 & & & 35 \\
H4 etched I & 25 & 68 & & & 68 \\
H4 etched II & 1 & 50 & & & 50 \\
S4a unetched & 100 & 51 & 0.18 & 100 & 0 \\
S4a etched II & 19 & 175 & 0.53 & 89 & 40 \\
S4a etched II & 5 & 308 & 0.80 & 76 & 364 \\
S4a hollow & 0 & 294 & & &
\end{tabular}

for cubes with a smooth surface. When we consider a smooth hematite cube with a density of $5.25 \mathrm{~g} \mathrm{~cm}^{-3}$ (ref. 43) and a surface area of $35 \mathrm{~m}^{2} \mathrm{~g}^{-1}$, we arrive at an edge length for that cube of $33 \mathrm{~nm}$. This large difference between the actual size of $920 \mathrm{~nm}$ demonstrates that the hematite cubes have a rough surface, which was already observed in high magnification SEM images (see Fig. S5 in the ESI†).

3.1.1.2 Silica coated hematite cubes. From the typical TEM images shown in Fig. 3, it is clear that hematite etching also occurs when the hematite core is enclosed by a silica coating. The distinct butterfly-like shape appears inside the silica box as the etching advances. If the hematite cores are sufficiently etched, they move around freely as a result of Brownian motion which can be visualized with optical microscopy. We refer to these particles with a mobile core enclosed by a silica box as rattles. A distinct advantage of these rattles over rattles prepared by the removal of a sacrificial middle-layer ('sandwich 


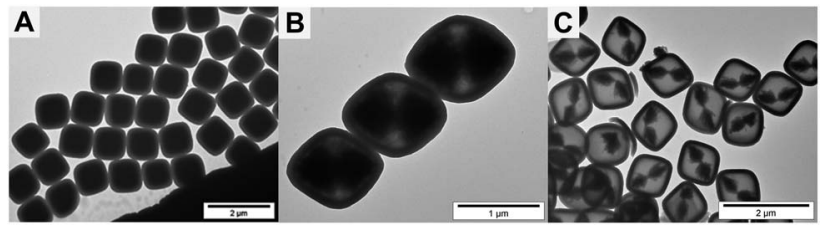

Fig. 3 Representative TEM images of silica coated hematite cubes (S4a) that have been partially hematite etched, referred to as rattles. The etching becomes increasingly visible when the amount of remaining hematite is decreased. (A) $100 \mathrm{wt} \%$ of the original amount of hematite present and (B) and (C) $19 \mathrm{wt} \%$ and $5 \mathrm{wt} \%$ remaining of the initial amount of hematite. When the cores are sufficiently etched, they can move freely inside the silica cube by Brownian motion.

method' $)^{2,9,11}$ is that the encapsulated particle can be manipulated directly; the desired shape and size can be simply obtained by selective etching of the core with hydrochloric acid.

Table 2 lists the total BET-surface areas of the rattles, which are considerably higher than those of the bare hematite particles. This is a result of the high porosity of the Stöber silica coating. The table also lists the contributions of the separate components of the silica coated particles, i.e., the contributions of the hematite core and the silica shell. These values were calculated by multiplying the component's mass fraction with its corresponding BET-surface area and comparing it with the total BET-surface area. As an example, we show that for unetched silica coated hematite cubes the surface area is basically determined by that of the silica shell: the mass fraction of silica is approximately 0.18 and the BET-surface area of hollow silica cubes is $294 \mathrm{~m}^{2} \mathrm{~g}^{-1}$, which yields a contribution of the silica shell of $53 \mathrm{~m}^{2}$. Since this nearly equals the BET-surface area of the entire silica coated hematite cube, it suggests that the silica shell entirely and fittingly covers the hematite core, and that the exposed hematite surface is roughly similar to the silica free surface lost due to the hematite/silica interface. Similar calculations for the rattles show that as hematite etching proceeds, the core detaches from the shell and its contribution to the total BET-surface area grows. We must note that the precise values highly depend on the mass fractions and assumed material densities $\left(5.25 \mathrm{~g} \mathrm{~cm}^{-3}\right.$ and $2.0 \mathrm{~g} \mathrm{~cm}^{-3}$ for hematite and silica, respectively), but the observed trend does not change. The resulting surface areas of the hematite cores are given in the last column of Table 2 .

\subsection{Degradation experiments}

\subsubsection{Dispersed cubes}

3.2.1.1 Regular laboratory lighting. From Fig. 4A it is clear that the degradation of methylene blue is indeed promoted by bare hematite cubes compared to the degradation by only hydrogen peroxide (the blank). Although an enhancement of the degradation process is observed, the observed rates are still much lower than that for homogenous Fenton catalysts, i.e., aqueous iron ion solutions, as is expected. From literature it is known that hematite is not the most efficient iron oxide for the modified Fenton reaction without active illumination and our observed degradation behavior is in line with previous reports. ${ }^{20-22}$
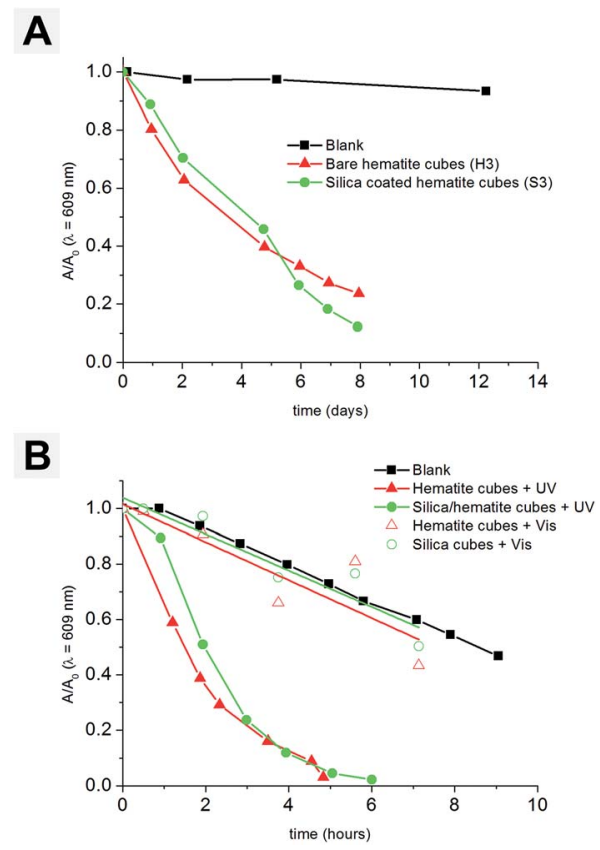

Fig. 4 Normalized absorbance of methylene blue at $\lambda=609 \mathrm{~nm}$ as a function of reaction time. The drawn lines are to guide the eye. (A) Compared to the blank (only hydrogen peroxide and methylene blue), the degradation of methylene blue in regular laboratory lighting is promoted by bare hematite cubes, as well as silica coated hematite cubes. The silica coating does not reduce the degradation rate at comparable hematite surface area. (B) Illumination with visible or UV light drastically lowers the degradation time from days to hours. Similar degradation rates for bare hematite and silica coated hematite cubes are found.

From Fig. 4A, it is clear that the degradation of methylene blue is not hampered by the silica coating shielding the hematite core: the rate of degradation by silica coated hematite cubes is comparable to that of bare hematite cubes having similar total surface areas of the core. This result was probably effected by the combination of the adsorption of both methylene blue and hydrogen peroxide to the silica surface, and the inherent acidity of the silica surface, as proposed in previous studies. $^{\mathbf{2 0 , 2 3 , 4 4 , 4 5}}$ The former brings the reactants closer to the hematite surface. The latter is beneficial since the Fenton reaction is enhanced at low $\mathrm{pH}^{23-25}$ Moreover, a change in chemical environment of the hematite core has been posed as a possible explanation of this positive effect of a silica matrix, ${ }^{19,21,23}$ e.g., less scavenging of the reactive hydroxyl radicals at the silica/iron oxide surface compared to the bare iron oxide surface or a change in the favored reactions in the reaction chain. Analogous results were obtained for the degradation of rhodamine B (see Fig. S7 in the ESI $\dagger$ ). In essence, the cubic shape of the particles can be maintained while the surface area of the core can be conveniently adjusted, i.e., a catalyst in a silica box. The accelerated degradation has been accomplished for three different batches of silica coated hematite cubes and for the rattles. The core can still be reached through a silica coating of $75 \mathrm{~nm}$ in thickness.

Interestingly, in the case of both the bare and the silica coated hematite cubes, the size, the amount of particles and the 
silica coating thickness did not influence the degradation rate (data shown in the ESI $\dagger$ ). From these results, together with the low, albeit significant, degradation speeds, we infer that the degradation reaction is primarily governed by other properties of the hematite cubes. Considering that hematite is a known photocatalyst, ${ }^{32,35,37,46,47}$ we investigated the influence of active illumination with visible and UV light.

3.2.1.2 Illumination with visible and UV light. When we actively illuminate the samples with visible or UV light, we observe a striking acceleration of the degradation of methylene blue (Fig. 4B). Complete decolorization is achieved on a time scale of hours instead of the two weeks needed without illumination. Furthermore, the silica coating covering the hematite core does again not impede the degradation reaction, despite its thickness of $65 \mathrm{~nm}$. This demonstrates that the porous silica coating is sufficiently transparent for the used light. Considering that the absorption coefficient of silica at the used wavelengths is negligible, we expect that the influence of the thickness of the silica coating is correspondingly negligible for the degradation rates. ${ }^{48}$

The increased degradation is effected by the generation of charge carriers upon illumination with light of wavelength shorter than the bandgap of hematite $\left(E_{\mathrm{g}}=2.2 \mathrm{eV}\right)$. The generated charge carriers can then be annihilated in various ways, such as by direct photo-oxidation of the dye; ${ }^{49}$ by the formation of hydroxyl radicals catalyzed by the hematite core, which initiates the Fenton reaction (photo-Fenton reaction); or by direct hydroxyl radical formation from hydrogen peroxide $^{24,25,35,37,47}$

$$
\mathrm{H}_{2} \mathrm{O}_{2}+h \nu \rightarrow 2 \mathrm{OH}^{\cdot}
$$

The measurements also show that UV light enhances the degradation more than visible light. A possible cause is the more efficient decomposition of hydrogen peroxide into hydroxyl radicals with UV light. ${ }^{24}$ Blank experiments with only hydrogen peroxide and methylene blue confirm that the dye is indeed degraded faster by UV light than by visible light (hours versus days).

3.2.2 Cubes on a substrate. Since cubes can form a theoretical packing of $100 \%$, assembling the catalytic cubes onto a substrate is appealing for many applications and also a facile approach for recovery of the particles. The cubes have been conveniently deposited onto mica substrates by evaporating the aqueous solvent rendering close-packed multilayer structures as displayed in Fig. 5. Such packings were obtained with both unetched and hematite etched cubic colloids.

Unfortunately, the cubes did not adhere sufficiently to the substrate and detached from the substrate during the experiments. Therefore, we explored the influence of heating on the attachment by varying the temperature from $200{ }^{\circ} \mathrm{C}$ to $450{ }^{\circ} \mathrm{C}$ and found that at increasing temperature of the heat treatment, the cubes remained better attached to the substrate. The appearance of the heat treated cubes was not different from untreated cubes. However, we did not achieve attachment of all deposited cubes; for the vertically hung substrates a maximum of $84 \%$ remained (heated at $450{ }^{\circ} \mathrm{C}$ ) and for the horizontally

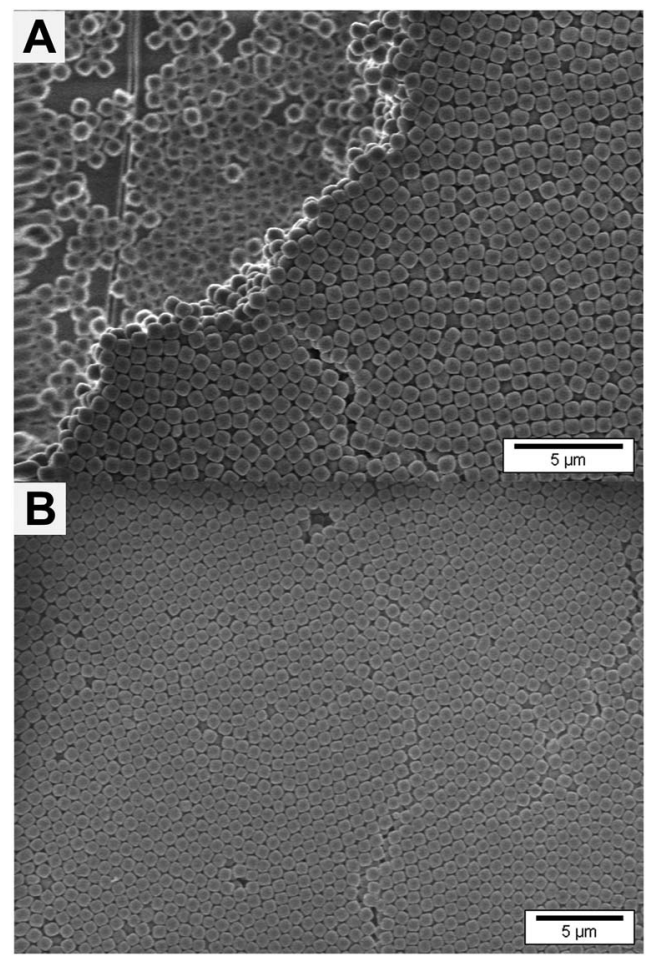

Fig. 5 Typical SEM images of close-packed multilayer structures formed from silica coated hematite cubes deposited on mica substrates. The cubes assembled by convection forces caused by the evaporation of the aqueous solvent.

placed substrate $81 \%$ (heated at $450{ }^{\circ} \mathrm{C}$ ). To overcome this inconvenience, more research is needed regarding a more suitable heat treatment and also a more suitable substrate. Mica has been chosen because it can be made atomically flat, but other materials might give better adherence. ${ }^{50}$ For instance, positively charged alumina can bind the silica cubes to the surface by electrostatic attraction and $\mathrm{Si}-\mathrm{O}-\mathrm{Al}$ bonds.

Nevertheless, the substrates with cubes could still be employed for degradation experiments and Fig. 6 illustrates the activity of the deposited cubes heated at $400{ }^{\circ} \mathrm{C}$. The degradation of methylene blue under regular laboratory lighting proceeds faster in the presence of cubes than with only an empty mica substrate in the reaction mixture, as was observed for both types of used setups. However, the degradation was slower for cubes on a substrate than dispersed cubes. We attribute this difference to the lower amount of cubes effectively available for the reaction. Although the total amount of deposited cubes is the same as or even larger than the amount for the dispersed cubes experiments, mainly the cubes in the top layer are accessible. Moreover, the deposited cubes cannot be approached from all sides, whereas for dispersed cubes all sides are equally exposed.

Although released cubes are assumed to cause a higher apparent degradation rate, we determined that the enhanced degradation by the substrates is not solely caused by detached cubes dispersed freely in the reaction mixture (compare $\boldsymbol{\Delta}$ with $\checkmark$ in Fig. 6). 


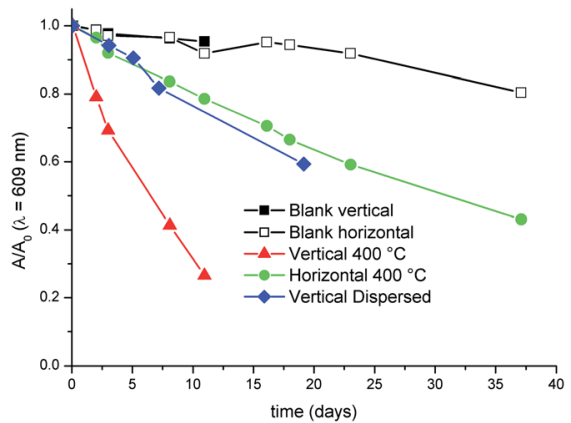

Fig. 6 Normalized absorbance of methylene blue at $\lambda=609 \mathrm{~nm}$ as a function of reaction time. The degradation of methylene blue is still promoted by silica coated hematite cubes (S4b) immobilized on a substrate compared to the blank (only hydrogen peroxide, methylene blue and an empty substrate). The experiments were conducted in regular laboratory lighting. Two different setups were used: vertically hanging substrate and horizontally placed substrate. The substrates were heated to $400{ }^{\circ} \mathrm{C}$ to improve attachment of the cubes. The same amount of dispersed cubes as detached cubes from the vertically hung substrate was used to determine the influence of free catalyst.

In view of recycling the particles by reusing the close-packed cubes on a substrate for a second degradation reaction, we established that the deposited cubes are still active and that a sufficient amount of cubes remained on the substrate for reuse, despite the detachment.

3.2.3 Particle stability. Since hematite is one of the most thermodynamically stable iron oxides, ${ }^{33,51}$ it is not expected to be affected by the degradation reaction itself. However, the reaction was conducted at acidic conditions (approximately $\mathrm{pH}$ 3 ), at which hematite is known to dissolve. Despite the low $\mathrm{pH}$ of the reaction mixture, we did not observe a drastic change in the morphology of the hematite cubic colloids after the reaction. If a substantial amount of hematite would dissolve to ferric ions, we expect the reaction mixture to become drastically more acidic due to the formation of an aqua acid from these ions, ${ }^{52}$ but the $\mathrm{pH}$ remained constant over time. Previous reports also show little leaching of iron ions from iron oxide particles. ${ }^{22,44,53}$

Unfortunately, we did observe a change in the silica coating around the hematite core after the degradation reaction. The TEM image in Fig. 7D is exemplary for silica coated hematite cubes after reaction with hydrogen peroxide and methylene blue without illumination. This damage to the silica coating was definitely not caused by the slow dissolution of silica in water, ${ }^{45}$ because we did not observe a change in the silica coating of cubes that have been stored in water at room temperature for at least a few months. Alternatively, we attribute the deterioration of the silica coating to pressure built up inside the coating. The increased pressure could have emerged from a rise in the osmotic pressure inside the silica coating due to the generated products of hydrogen peroxide decomposition, creating a flow of solvent towards the silica coating. However, hydrogen peroxide alone did not cause the large extent of damage as seen in Fig. 7D, although some breakage did occur. Therefore, the deterioration of the silica coating is believed to be mainly induced by the modified Fenton reaction, i.e., the combination

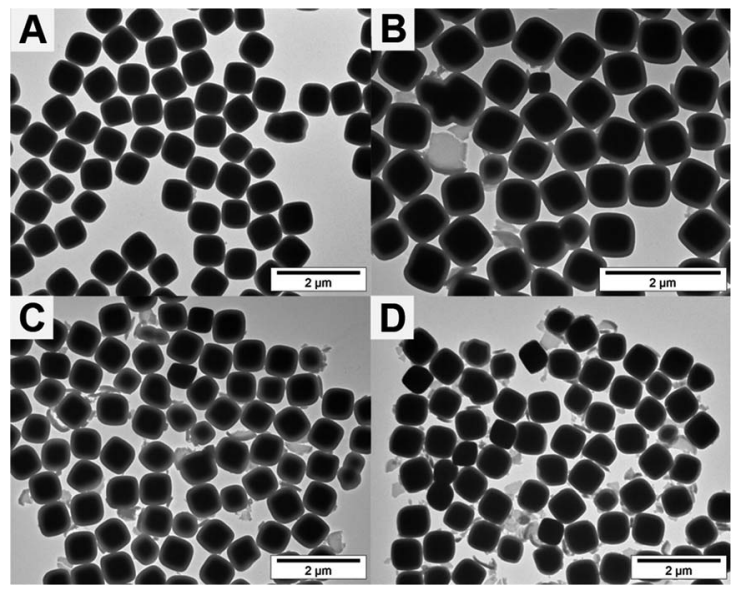

Fig. 7 Typical TEM images of silica coated hematite cubes (coating thickness $80 \mathrm{~nm}$ ) dispersed in water, hydrogen peroxide and methylene blue followed in time. The deterioration of the silica coating did not occur instantaneously, but gradually over reaction time: (A) a few hours, $0 \%$ broken silica coatings; (B) three days, 2\% broken silica coatings; (C) one week, 7\% broken silica coatings; and (D) two weeks, $11 \%$ broken silica coatings.

of the degradation reaction and hydrogen peroxide decomposition. The degradation reaction generates oxygen gas, degradation products which could contribute to an increased osmotic pressure, and eventually even carbon dioxide gas. ${ }^{18,19,24}$ Indeed, gas bubbles in the reaction mixture were observed. We monitored the stability of the silica coated cubes in reaction over time and noted that the breakage of the silica coating is not instantaneous but rather gradual. The amount of cubes that have a broken silica coating or are entirely bare increases from $0 \%$ for cubes after a few hours of reaction to $11 \%$ after two weeks of reaction (Fig. 7). Comparable numbers were found for cubes with a coating thickness of approximately $80 \mathrm{~nm}$ and $100 \mathrm{~nm}$. Admittedly, reduced mechanical stability of very thin hollow silica shells of $20 \mathrm{~nm}$ have previously been observed. ${ }^{17}$ Therefore, the use of thicker silica coatings is more appropriate for the application of the core-shell cubes in this degradation reaction.

Nevertheless, the stability of the coatings was enhanced by thermally treating the silica coated hematite. Although the silica coatings of the heated cubes were still affected by the reaction, less broken coatings were observed compared to the freely dispersed cubes. Furthermore, UV illumination also appeared to be beneficial for the stability of the silica coatings, since less broken coatings were observed after the degradation reaction than without UV illumination (7\% of broken silica coatings, after full degradation). The advantageous effect probably originates from one of the sources of the increased degradation rate, namely the degradation of hydrogen peroxide to hydroxyl radicals under influence of UV light (reaction VI). Due to this additional path for radical formation, the amount of hydroxyl radicals formed at the catalyst surface is lessened and, consequently, the pressure inside the silica coatings decreases.

Notwithstanding the observation of some broken and damaged silica coatings, it should be noted that the majority of 
cubes were still coated by a layer of silica, i.e., the catalyst in a silica box was maintained.

\section{Conclusions}

In conclusion, we have demonstrated the concept of functional colloidal cubes for the enhanced degradation of organic dyes in a modified Fenton reaction. The advantage of these cubes is twofold. On the one hand, the cubic shape of these core-shell $\alpha$ $\mathrm{Fe}_{2} \mathrm{O}_{3} @ \mathrm{SiO}_{2}$ particles allows for the formation of dense assemblies supported by a substrate. On the other hand, the hematite core acts as an accelerator for the degradation of methylene blue and rhodamine $\mathrm{B}$ in the presence of hydrogen peroxide. The degradation reaction is further promoted by illumination with visible or UV light. The permeable silica shell does not hinder this degradation enhancement by the hematite core, since comparable degradation rates for either were observed. Consequently, the cubic shape for close-packed assemblies can be preserved, while rattles can be prepared leading to modified surface areas of the hematite core. The cubic colloids retain their degradation functionality when densely packed onto a substrate.

For the future, optimization of this system is needed for practical applications. Additionally, degradation experiments with the rattle-like cubes could give more insight into the catalytic activity of the hematite core as a function of the surface area. Moreover, further research could focus on expanding the collection of functional materials combined with the silica cubes, e.g., metal nanoparticles. Unlike the system presented in this work, however, incorporating these functional materials requires methods to synthesize them inside a hollow silica cube.

\section{Acknowledgements}

We are grateful to Dr Ana-Mariana Ürmenyí, who introduced us to the Fenton reaction. We would also like to thank Mr Ad Mens (Inorganic Chemistry and Catalysis, Utrecht University) for the nitrogen physisorption measurements and Professor John Kelly for his input. Petra de Jongh acknowledges the NRSCC Solar Fuels program. This research is supported by the Dutch Technology Foundation STW, Netherlands under the partnership program STW-Hyflux CEPAration Inorganic and Hybrid Membranes. We thank STW and Hyflux for their financial support.

\section{References}

1 S. Joo, J. Park, C. Tsung, Y. Yamada, P. Yang and G. Somorjai, Nat. Mater., 2009, 8, 126-131.

2 M. Kim, K. Sohn, H. B. Na and T. Hyeon, Nano Lett., 2002, 2, 1383-1387.

3 Y. Deng, Y. Cai, Z. Sun, J. Liu, C. Liu, J. Wei, W. Li, C. Liu, Y. Wang and D. Zhao, J. Am. Chem. Soc., 2010, 132, 84668473.

4 C. Huang, W. Huang and C. Yeh, Biomaterials, 2011, 32, 556564.
5 C. Graf, D. Vossen, A. Imhof and A. van Blaaderen, Langmuir, 2003, 19, 6693-6700.

6 K. Chou and C. Chen, Microporous Mesoporous Mater., 2007, 98, 208-213.

7 V. Calderone, J. Schuetz-Widoniak, G. Bezemer, G. Bakker, C. Steurs and A. Philipse, Catal. Lett., 2010, 137, 132-140.

8 J. Kim, S. Yoon and J. Yu, Chem. Commun., 2003, 790-791.

9 K. Kamata, Y. Lu and Y. Xia, J. Am. Chem. Soc., 2003, 125, 2384-2385.

10 J. Lee, J. Park and H. Song, Adv. Mater., 2008, 20, 1523-1528. 11 X. Huang, C. Guo, L. Zuo, N. Zheng and G. Stucky, Small, 2009, 5, 361-365.

12 P. Arnal, M. Comotti and F. Schueth, Angew. Chem., Int. Ed., 2006, 45, 8224-8227.

13 L. Rossi, S. Sacanna, W. Irvine, P. Chaikin, D. Pine and A. Philipse, Soft Matter, 2011, 7, 4139-4142.

14 J. Meijer, F. Hagemans, L. Rossi, D. Byelov, S. Castillo, A. Snigirev, I. Snigireva, A. Philipse and A. Petukhov, Langmuir, 2012, 28, 7631-7638.

15 R. Batten, F. Stillinger and S. Torquato, Phys. Rev. E: Stat., Nonlinear, Soft Matter Phys., 2010, 81, 061105.

16 Y. Jiao, F. H. Stillinger and S. Torquato, Phys. Rev. E: Stat., Nonlinear, Soft Matter Phys., 2010, 81, 041304.

17 S. I. R. Castillo, S. Ouhajji, S. Fokker, B. H. Erné, C. T. W. M. Schneijdenberg, D. M. E. Thies-Weesie and A. P. Philipse, Microporous Mesoporous Mater., 2014, 195, 75-86.

18 P. Bautista, A. Mohedano, J. Casas, J. Zazo and J. Rodriguez, J. Chem. Technol. Biotechnol., 2008, 83, 1323-1338.

19 E. Neyens and J. Baeyens, J. Hazard. Mater., 2003, 98, 33-50.

20 Z. Cui, Z. Chen, C. Cao, L. Jiang and W. Song, Chem. Commun., 2013, 49, 2332-2334.

21 H. Lim, J. Lee, S. Jin, J. Kim, J. Yoon and T. Hyeon, Chem. Commun., 2006, 463-465.

22 S. Lee, J. Oh and Y. Park, Bull. Korean Chem. Soc., 2006, 27, 489-494.

23 A. Pham, C. Lee, F. Doyle and D. Sedlak, Environ. Sci. Technol., 2009, 43, 8930-8935.

24 E. Brillas, I. Sires and M. Oturan, Chem. Rev., 2009, 109, 6570-6631.

25 M. Hartmann, S. Kullmann and H. Keller, J. Mater. Chem., 2010, 20, 9002-9017.

26 W. Barb, J. Baxendale, P. George and K. Harggrave, Trans. Faraday Soc., 1951, 47, 462-500.

27 W. Barb, J. Baxendale, P. George and K. Harggrave, Trans. Faraday Soc., 1951, 47, 591-616.

28 S. Navalon, M. Alvaro and H. Garcia, Appl. Catal., B, 2010, 99, 1-26.

29 A. Kay, I. Cesar and M. Graetzel, J. Am. Chem. Soc., 2006, 128, 15714-15721.

30 I. Cesar, A. Kay, J. Martinez and M. Graetzel, J. Am. Chem. Soc., 2006, 128, 4582-4583.

31 K. Young, B. Klahr, O. Zandi and T. Hamann, Catal. Sci. Technol., 2013, 3, 1660-1671.

32 C. Du, X. Yang, M. Mayer, H. Hoyt, J. Xie, G. McMahon, G. Bischoping and D. Wang, Angew. Chem., Int. Ed., 2013, 52, 12692-12695. 
33 R. Cornell and U. Schwertmann, The iron oxides: structure, properties, reactions, occurrences and uses, Wiley-VCH, 2003.

34 B. Xu, B. Huang, H. Cheng, Z. Wang, X. Qin, X. Zhang and Y. Dai, Chem. Commun., 2012, 48, 6529-6531.

35 Y. Zhao, F. Pan, H. Li, T. Niu, G. Xu and W. Chen, J. Mater. Chem. A, 2013, 1, 7242-7246.

36 X. Zhou, Q. Xu, W. Lei, T. Zhang, X. Qi, G. Liu, K. Deng and J. Yu, Small, 2014, 10, 674-679.

37 X. Zhou, H. Yang, C. Wang, X. Mao, Y. Wang, Y. Yang and G. Liu, J. Phys. Chem. C, 2010, 114, 17051-17061.

38 T. Sugimoto and K. Sakata, J. Colloid Interface Sci., 1992, 152, 587-590.

39 T. Sugimoto, K. Sakata and A. Muramatsu, J. Colloid Interface Sci., 1993, 159, 372-382.

40 J. S. Falcone Jr., J. L. Bass, P. H. Krumrine, K. Brensinger and E. R. Schenk, J. Phys. Chem. A, 2010, 114, 2438-2446.

41 J. Osswald and K. Fehr, J. Mater. Sci., 2006, 41, 1335-1339.

42 L. Rossi, PhD thesis, Universiteit Utrecht, 2012.

43 R. Perry and D. Green, Perry's Chemical Engineers' Handbook, McGraw-Hill, 1984.

44 N. Ferroudj, J. Nzimoto, A. Davidson, D. Talbot, E. Briot, V. Dupuis, A. Bée, M. S. Medjram and S. Abramson, Appl. Catal., B, 2013, 136-137, 9-18.
45 R. Iler, The chemistry of silica. Solubility, polymerization, colloid and surface properties, and biochemistry, John Wiley \& Sons, Inc., 1979.

46 W. Du, Y. Xu and Y. Wang, Langmuir, 2008, 24, 175-181.

47 X. Zhou, J. Lan, G. Liu, K. Deng, Y. Yang, G. Nie, J. Yu and L. Zhi, Angew. Chem., Int. Ed., 2012, 51, 178-182.

48 R. Kitamura, L. Pilon and M. Jonasz, Appl. Opt., 2007, 46, 8118-8133.

49 J.-Z. Kong, A.-D. Li, X.-Y. Li, H.-F. Zhai, W.-Q. Zhang, Y.-P. Gong, H. Li and D. Wu, J. Solid State Chem., 2010, 183, 1359-1364.

50 Inorganic Membranes. Synthesis, characterization and applications, ed. R. Mallada and M. Menéndez, Elsevier, 2008.

$51 \mathrm{U}$. Schwertmann and R. Cornell, Iron Oxides in the Laboratory. Preparation and Characterization, Wiley-VCH, 2000.

52 D. Harris, Quantitative chemical analysis, seventh edition, WH Freeman, 2007.

53 N. Panda, H. Sahoo and S. Mohapatra, J. Hazard. Mater., 2011, 185, 359-365. 